\title{
Kreatin beschleunigt das SSRI-Ansprechen
}

Fragestellung: Beschleunigt und verstärkt die Augmentationsbehandlung mit Kreatinmonohydrat die Response auf eine SSRI-Behandlung bei Frauen mit schwerer depressiver Episode?

Hintergrund: Frühere Studien weisen darauf hin, dass die Gabe von Kreatin die zerebrale Verfügbarkeit von Phosphokreatin erhöht. Dies könnte zu einer Veränderung der Kreatinkinaseaktivität und zu einer Erhöhung von Adenosintriphosphat (ATP) beitragen. Eine präklinische Studie wies auf eine selektive Wirksamkeit bei weiblichen Nagetieren hin. Diese geschlechtsabhängigen Effekte könnten durch eine östrogenabhängige Modulation der mitochondrialen Funktion vermittelt werden.

Patienten und Methodik: 52 Frauen mit schwerer depressiver Episode gemäß DSM-IV (Alter 19 bis 65 Jahre, 17-Item-HAMDScore $\geq 16$, mindestens acht Wochen vor Studie medikamentenfrei, Ausschluss anderer schwerer Erkrankungen) wurden in eine achtwöchige, doppelblinde und placebokontrollierte klinische Studie eingeschlossen und erhielten zusätzlich zu Escitalopram (10 mg/d über eine Woche, individuelle Dosierung innerhalb der weiteren Wochen) entweder Kreatin ( $5 \mathrm{~g} / \mathrm{d}, \mathrm{n}=25)$ oder Placebo $(n=27)$. Eine limitierte zusätzliche Gabe von Lorazepam oder Zolpidem war erlaubt. Haupt-Outcome-Parameter waren die Response (> 50\% Reduktion des HAMD-17-Scores) und Remission (HAMD-17-Score $\leq 7$ ) in Woche 8.

Ergebnisse: Im Vergleich zur Placebo- zeigte die Verumgruppe eine signifikant höhere Verbesserung im Hamilton-Score nach zwei Wochen. Die differenzielle Response war über vier und acht Wochen erhalten. 13 (52,0\%) der Patienten mit Kreatin-Augmentation und sieben $(25,9 \%)$ Patienten in der Placebogruppe zeigten eine Remission in Woche 8 (OR 6,92, p = 0,008). Es wurden keine signifikanten Unterschiede bezüglich Studienabbrecher $(8$ Kreatin, 5 Placebo) oder in der Häufigkeit unerwünschter Wirkungen berichtet (Kreatin 36 Ereignisse; Placebo 45 Ereignisse). Die mittlere Escitalopram-Dosierung war in der Kreatingruppe (15,0 mg/d) und der Placebogruppe (15,7 mg/d). Eine Einnahme von Lorazepam wurde bei zehn Patienten mit Kreatin-Augmentation und zwölf Patienten mit Placeboaugmentation berichtet. Zolpidem wurde bei sechs Patienten in der Kreatin- und neun Patienten in der Placebogruppe verschrieben. Die Gruppen unterschieden sich nicht in relevanten demografischen Variablen oder klinischen Variablen, einschließlich Beginn der depressiven

Lyoo K, Yoon S, Kim TS et al. A randomized, double-blind placebo-controlled trial of oral creatine monohydrate augmentation for enhanced response to a selective serotonin reuptake inhibitor in wom-en with major depressive disorder. Am J

Psychiatry 2012; 169: 937-45
Störung, Schwere der aktuellen depressiven Episode, der Episodenhäufigkeit oder der Vorbehandlung. Die Raten unerwünschter Wirkungen waren in beiden Gruppen vergleichbar, schwere unerwünschte Wirkungen traten nicht auf. Keine Unterschiede in den Laborparametern oder BMI wurden beobachtet.
Schlussfolgerung: Die Autoren folgern, dass die KreatinAugmentation bei SSRI-Behandlung zu einer rascheren und effektiveren Wirksamkeit bei Frauen mit Major-Depression führt.

\section{-Kommentar von Christoph Nissen, Freiburg Mehr Untersuchungen notwendig}

In einer interessanten Proof-of-concept-Studie legen die Autoren erstmals Daten vor, dass eine Augmentation mit Kreatin die Wirksamkeit einer SSRI-Behandlung, zumindest bei Frauen mit schwerer depressiver Episode, erhöht (JADAD-Score 5). Es handelt sich um die erste klinische Studie in einer relativ kleinen Stichprobe, so dass weitere Untersuchungen zur Wirksamkeit und Sicherheit notwendig sind. Die Autoren führen zwar präklinische Befunde für eine mögliche präferenzielle Wirksamkeit von Kreatin bei Frauen an. Das Postulat eines geschlechtsabhängigen Dimorphismus in einem so basalen Stoffwechselweg wie der ATP-Gewinnung überrascht jedoch. Aber auch unabhängig von einem möglichen Geschlechtsdimorphismus sind weitere Untersuchungen notwendig, um den neuronalen Mechanismus weiter aufzuklären. Zu beachten ist, dass Pilotdaten auf einen erhöhten Switch in eine manische Episode hinweisen [1]. Relevante Gesundheitsrisiken durch das Nahrungsergänzungsmittel Kreatin sind nicht bekannt. Bei dauerhafter Einnahme von hohen Dosierungen (10-20 g/d) wurden milde unerwünschte Wirkungen (Gewichtszunahme, Muskelkrämpfe, gastrointestinale Beschwerden) berichtet.

Vor dem Hintergrund, dass depressive Störungen die weltweit führende Ursache für eine eingeschränkte Lebensqualität sind, zeigt die Studie einen interessanten neuen Therapieansatz auf. Weitere Untersuchungen sind notwendig, um zu prüfen, inwieweit sich dieser Ansatz in der breiteren klinischen Anwendung etablieren kann.

Referenz

1. Roitman S et al. Bipolar Disord 2007; 9: 754-8

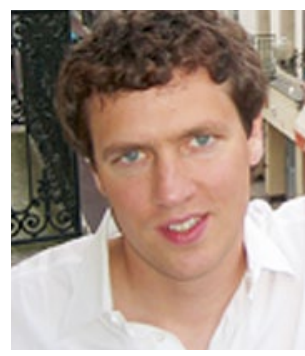

PD Dr. med. Christoph Nissen, Freiburg i. Br.

Oberarzt; Leitung der AG Neurophysiologie, Universitätsklinikum Freiburg E-Mail: christoph.nissen@uniklinikfreiburg.de 